\title{
- Smart Medical Self-diagnostic Tools
}

\section{Aparna Beena Unni}

\section{IJCRR}

Section: Healthcare

ISI Impact Factor

(2019-20): 1.628

IC Value (2019): 90.81

SJIF (2020) $=7.893$

Silesian Centerfor Education and Interdisciplinary Research (SMCEBI), 75 Pulku Piechoty 1a, 41-50o Chorzow, Poland.

E-mail: aparna.beena-unni@smcebiedu.pl

\section{SMART MEDICAL TOOLS}

Smart medical equipment or thinking, self-determining diagnostic tools is for diagnoses and treatments. Today this is not science fiction. Many such intelligent medical devices are transforming modern medicine.

The last few decades have seen a very revolutionary change in the tone of modern medicine. The main reason behind this is the advances in science and technology, which have been incorporated into the manufacture of medical devices that aid in diagnosis and treatment. A cardiograph recorded by an ECG machine is self-analyzed to diagnose the patient's heart failure accurately. The "intelligent" mammography machine that can predict breast cancer risk, the CT / MRI machines that tell if there is a risk of brain tumor cancer, and the robotic surgery machines that can only detect cancer cells and perform surgery are a reality today.

Modern automated decision-making devices, such as the driverless self-adhesive car, the computer that writes poetry and draws pictures, and the ability to make self-conscious decisions, are making great strides in the field of diagnosis and treatment today. This is possible because artificial intelligence can be embedded in such medical machines. Big data, advanced machine learning algorithms, and the advent of high-speed and efficient computers have given birth to these thinking machines.

In 2016, researchers at Google developed a deep learning algorithm that could detect diabetic retinopathy from images captured by a camera (a device used to detect glaucoma and multiple sclerosis eye diseases). Today, this system is widely used to screen diabetic retinopathy with the help of an experienced ophthalmologist. This artificial intelligence, which is often undetectable by human limitations, can even accurately predict the likelihood of developing the disease in the near future.

The use of artificial intelligence has made a big difference in the field of radiology imaging. AI systems are used in the field of diagnostics (Eg: Automated Imaging Analytics En- gine), which are capable of diagnosing X-ray / CT / MRI images without the assistance of a radiologist. But it is a matter of ethics to run a machine for complete diagnosis without human intervention. Therefore, such systems are usually used as a second opinion to help the doctor in their work and speed up. At the same time, such systems overcome the human error that can occur to a doctor. Such artificial intelligence systems can detect many abnormalities on an X-ray that cannot be detected by the human eye and can predict disease risks. Today, many countries are making extensive use of built-in intelligence for the diagnosis of tuberculosis and mass screening. Experts testify that this type of diagnosis has 99\% accuracy, which goes beyond human limitations.

IBM Watson Health and Google Health / Deep mind have made great strides in this area. In addition, some Indian companies have established themselves in this field. Radiologists are one of the scarcest medical disciplines in the world. In many remote areas of our country, it is often impossible to get such skilled doctors' services. The built-in intelligencebased radiology can also be a great solution to such difficulties, provided a person can monitor the proceedings.

\section{PATHOLOGY AI SYSTEM}

The Pathology AI system is widely used today in the diagnosis of cancer. Pathologists rely on microscopic examination of the slides for diagnosis. But it is natural for many experts to have different opinions on the same slide. However, the AI Pathology system can accurately diagnose a tumor cell faster than an expert pathologist and can detect the present stage of cancer (Predict Prognosis of stage). This ability is beyond the reach of the human eye.

There is another great possibility. An efficient algorithm makes an accurate diagnosis by providing medical knowledge and patient physical examination information (patients' symptoms, vital signs, blood test, etc.) in a large database. Experts testify that its efficiency is more than $20 \%$ better than that of a highly experienced general practitioner. This is a very 
modern achievement, but such a possibility was introduced in 1975. Edward Shortliffe and Bruce G Buchanan invented the Rule-based expert system. But today, it has been transformed into a highly efficient virtual doctor with the help of artificial intelligence. It will revolutionize the field of telemedicine. Today, the most widely used home care medical devices (Wearables and point of care equipments) are highly effective telemedicine systems that incorporate built-in intelligence. It is safe to stay at home and seek professional help, especially without having to go to the hospital.

This kind of artificial intelligence system is not meant to eliminate the doctor completely, but it is a second opinion system that helps the doctor in the diagnosis. A doctor makes a diagnosis based on the patient's symptoms (Vital sign, Lab reports). However, the AI system (Rule Based Algorithm / Machine Learning Algorithm's) allows the doctor to make an accurate diagnosis while the doctor using an AI Integrative decision support system provides the patient's test information to the AI system. Even a doctor with little or no experience but with good theoretical knowledge can make an accurate diagnosis.

\section{AI-BASED PRECISION MEDICINE}

Precision Medicine is another area where artificial intelligence is used. AI-based insulin pump is available today that provides the required dose of insulin to measure the body's glucose level each time, depending on the specific nature of the disease in each diabetic and his/her physical characteristics. AI-based precision medicine is a growing branch of medicine today that treats every patient according to their varying physical characteristics. Thus, the advancement of artificial intelligence has brought about profound changes in the field of diagnostic treatment. Only a few of them are mentioned here. Constructed systems are able to overcome human limitations in diagnosis and treatment. There is no doubt that these self-thinking Smart and Intelligent Medical Equipments will bring great strides in our health care.

\section{REFERENCE}

1. Aljurayfani M, Alghernas S, Shargabi A. Medical self-diagnostic system using artificial neural networks. In: 2019 International Conference on Computer and Information Sciences (ICCIS). IEEE. 2019 (pp. 1-5). 